\title{
CONCEPTION DES DISPOSITIFS DE FRANCHISSEMENTS POUR LA FAUNE AMPHIDROME (CREVETTES ET POISSONS) DES COURS D'EAU ANTILLAIS : UNE REVUE.
}

\author{
E. FIÈVET (1), A.L. ROUX (1), L. REDAUD (2) et J.M. SÉRANDOUR (3)
}

(1) U.M.R. C.N.R.S. n 5023, Écologie des Hydrosystèmes Fluviaux, Université Claude Bernard Lyon I, 69622 Villeurbanne Cedex, France. E-mail : efievet@ecolstat.univlyon1.fr.

(2) Parc National de la Guadeloupe, Service Connaissance et Gestion des Milieux Naturels, B.P. 13, Montéran, 97120 Saint-Claude, Guadeloupe. E-mail : Iredaud@parcnational.gp

(3) Direction Régionale de l'Environnement (DIREN), Service Eau, Milieux Aquatiques et Déchets, B.P. 105, allée des Lauriers - Circonvallation, 97120 Basse-Terre Cedex, Guadeloupe. E-mail : semad971@outremer.com

\section{RÉSUMÉ}

La macrofaune des cours d'eau antillais est constituée principalement d'espèces amphidromes de crevettes d'eau douce (Palaemonidae, Atyidae et Xiphocarididae) et de poissons (Gobiidae). Si la croissance et la reproduction de ces espèces ont lieu en eau douce, le développement larvaire nécessite divers degrés de salinité. La construction de prises d'eau (seuils et barrages collinaires) est susceptible de nuire à la fois aux migrations de dévalaison et aux migrations d'avalaison. Les migrations de dévalaison sont entreprises par les larves nouvellement nées qui sont emportées vers les estuaires par le courant. Les prises d'eau induisent donc des taux élevés de mortalité larvaire par entraînement des larves dans les conduites forcées. Les migrations d'avalaison sont principalement le fait de juvéniles qui présentent un rhéotactisme positif et un potentiel d'escalade des obstacles supérieurs à ceux des adultes. Les ouvrages de faible hauteur réalisés au travers des cours d'eau représentent des obstacles qui sont généralement franchis par escalade. Certains barrages collinaires réalisés aux Antilles ont été équipés de dispositifs de franchissements. Aucun critère biologique n'a cependant été pris en compte pour véritablement faciliter les migrations d'avalaison. En outre les migrations de dévalaison par dérive ont été ignorées. Les exigences d'une libre circulation en relation avec le cycle de vie amphidrome des espèces sont ici rappelées. Les taux élevés de mortalité larvaire peuvent être réduits en évitant les prélèvements d'eau durant les pics maximums de dérive qui ont lieu durant les premières heures après la tombée de la nuit. En cas de prise d'eau en continu des dispositifs lumineux sont susceptibles d'orienter les larves vers les dispositifs délivrant les débits réservés; ces dispositifs limiteraient ainsi l'entraînement des larves dans les conduites forcées. L'imperméabilité des barrages vis-à-vis des migrations d'avalaison peut disparaître complètement lorsque les ouvrages comportent des dispositifs sommaires de franchissements - de types " cascade " ou "passe en écharpe".

Mots-clés : Amphidromie, barrage, crevette d'eau douce, poisson, migration, passe à poissons, rivière insulaire tropicale, Antilles. 


\title{
CONCEPTION OF PASSAGE FACILITIES \\ FOR THE AMPHIDROMOUS BIOTA (FRESHWATER SHRIMPS AND FISHES) \\ OF THE WEST INDIES : A REVIEW.
}

\begin{abstract}
Stream macrobiota of the West Indies are dominated by amphidromous species of freshwater shrimps or prawns (Palaemonidae, Atyidae, and Xiphocarididae) and fishes (Gobiidae). If the growth and the reproduction of these species occur in fresh waters, their larval development needs salt water. Weirs and low-head dams for water abstraction may have impacts on both downstream and upstream migrations. Downstream migrations are performed out by newly hatched larvae which are swept by the water flow towards the sea. Water abstraction may induce a high entrainment mortality of larvae. Upstream migrations are carried out mainly by juveniles which present both higher positive rheotactic responses and higher climbing potentialities than adults. Juvenile individuals may have to get over the man-made barriers by climbing. Basic fishways were built on several occasions in the West Indies but no biotic criteria were really taken into account to facilitate the upstream migrations. Moreover the downstream migrations of the larvae were not taken into account. The necessity of maintening a free dam clearing in relation both to the life cycles and to the continuous reproduction of the species is recalled here. The entrainment mortality of larvae can be largely reduced if water is not abstracted during downstream migration which occurs mainly during the first hours after dusk. If water is abstracted continuously, light beams can be used to direct the larvae towards the compensation flows rather than towards the gauged flows. The imperviousnesses of low-head dams to upstream migrants can be eliminated by basic passage facilities such as " cascade-like passes " or "cross-side passes".
\end{abstract}

Key-words : Amphidromy, dam, freshwater shrimp, fish, migration, fishway, tropical insular stream, West Indies.

\section{INTRODUCTION}

Les Antilles correspondent à un chapelet d'îles comprenant au Nord les Grandes Antilles (Cuba, Jamaïque, Hispaniola et Puerto Rico) et au Sud les Petites Antilles, soit une vingtaine d'îles principales. Ces îles matérialisent la limite Est de la Mer des Caraïbes. Les Grandes Antilles et les plus grandes îles des Petites Antilles sont principalement constituées de zones montagneuses qui sont recouvertes, selon l'altitude, par différents types de forêts ombrophiles. Bien que tropicale, la faune de ces îles est considérée par la plupart des biogéographes comme très pauvre (LIEBHERR, 1988). Cette pauvreté a été vérifiée notamment sur la faune des cours d'eau (BAUCHOT, 1959 ; STARMÜLHNER et THÉRÉZIEN, 1982a et $b$; LIM et al., 1997). En effet “ l'accès aux rivières des îles océaniques est particulièrement difficile pour les espèces continentales, à l'exception des espèces euryhalines » (GILLET, 1983). Aussi les cours d'eau antillais sont dominés par des espèces amphidromes de poissons, de crevettes d'eau douce et de gastéropodes (STARMÜLHNER et THÉRÉZIEN, 1982a et b ; COVICH et MCDOWELL, 1996).

La reproduction des espèces amphidromes a lieu en eau douce, les larves migrent vers les milieux saumâtres ou marins, et les juvéniles remontent en eau douce (MCDOWALL, 1998). Les crevettes d'eau douce sont représentées aux Antilles par trois familles : Palaemonidae, Atyidae et Xiphocarididae (CHACE et HOBBS, 1969 ; CHACE, 1992). Des représentants de ces trois familles peuvent être rencontrés suffisamment en altitude pour que leur migration soit interrompue par l'édification de barrages (GILLET, 1983 ; HUNTE, 1978). Parmi les poissons, c'est le cas des gobies (Sicydium spp.) (GILLET, 1983 ; LIM et al., 1997). Le cas des gastéropodes ne sera pas pris en considération ici. 
Pour maintenir les populations d'amont, certains barrages ont été équipés de dispositifs de franchissements devant permettre la migration d'avalaison. Faute d'informations directement disponibles, aucun critère biologique n'a cependant été pris en compte lors de la conception de ces dispositifs (FIĖVET, 2000). En outre la migration de dévalaison des larves a largement été ignorée (BENSTEAD et al., 1999). Un exposé synoptique présentant le cycle biologique des espèces en relation avec les exigences spatiales et temporelles de libre circulation est proposé ici avant un bilan des connaissances biologiques à prendre en compte lors de la conception de dispositifs de franchissements.

\section{INTÉRÊTS DE LA CONSERVATION DES ESPÈCES CONSIDÉRÉES}

Si un ouvrage réalisé sur un cours d'eau devrait permettre en permanence la libre circulation des espèces, il est généralement admis que seuls certains types de déplacements doivent absolument être préservés. II s'agit de ceux qui, de près ou de loin, sont liés à la reproduction des espèces menacées ou présentant une importance socio-économique (PORCHER et TRAVADE, 1992). Si aucune espèce dulcicole n'a été reconnue immédiatement menacée aux Antilles, les crevettes d'eau douce, notamment les plus grandes espèces de Macrobrachium et d'Atya, font l'objet d'une pêche artisanale (CHACE et HOBBS, 1969 ; GRÉGOIRE, 1991). Limportance de cette pêche - nombre de pêcheurs, quantités prélevées, vente du produit de la pêche - est cependant très peu connue. La raréfaction des grandes crevettes (Ouassous) fort appréciées sur le plan culinaire a entraîné la recherche d'espèces de substitution, d'où les importations et l'élevage de Macrobrachium rosenbergii, espèce asiatique très consommée aux Antilles (HERMAN et al., 1999). Les juvéniles de Sicydium associées aux alevins d'autres poissons amphidromes font l'objet d'une pêche en estuaire lors de leur remontée massive des rivières (BELL et BROWN, 1995 ; FIEVEET et Le GUENNEC, 1998). Cette pêche serait davantage pratiquée dans les îles anglophones (Dominique, Sainte Lucie...) qu'aux Antilles françaises (Guadeloupe et Martinique).

Outre son importance socio-économique, la faune amphidrome joue un rôle majeur dans le fonctionnement des écosystèmes aquatiques antillais. Les chaînes alimentaires y sont en effet très courtes et la faune amphidrome représente la majorité de la biomasse (COVICH et MCDOWELL, 1996 ; PRINGLE, 1997). De part leurs diverses activités (bioturbation, alimentation, déplacements), certaines crevettes et certains poissons ont un effet spectaculaire sur la biomasse d'algue, sur la structure des communautés notamment celles d'insectes ainsi que sur le transport solide des particules fines (rôle d'organismes ingénieurs sensus JONES et al., 1994 ; STATZNER et al., 2000) (CHACE et HOBBS, 1969 ; PRINGLE et al., 1993 ; PRINGLE et BLAKE, 1994 ; PRINGLE, 1996 ; PRINGLE et HAMAZAKI, 1997). La conservation de la faune amphidrome en amont des ouvrages est donc essentielle pour garantir l'intégrité des écosystèmes aquatiques (PRINGLE et SCATENA, 1999a), gage d'une eau de bonne qualité (YOUNG et al., 1994). En outre les larves qui rejoignent en grande quantité les estuaires (BELL et BROWN, 1995 ; HOLMQUIST et al., 1998 ; BENSTEAD et al., 1999) constituent une manne pour les poissons marins dont les juvéniles fréquentent les estuaires voire des zones d'eau douce pour certains (HOSTACHE, 1992) (par exemples Soles, Carangues, Mulets ; E. FIĖVET, données non publiées).

\section{CYCLE BIOLOGIQUE ET EXIGENCES DE LIBRE CIRCULATION}

\section{Reproduction}

Un résumé du cycle biologique des crevettes d'eau douce et des gobies (Sicydium spp.) des Antilles est proposé par la Figure 1. La reproduction des crevettes et des gobies lieu en eau douce tandis que le développement larvaire se déroule en mer (Figure 1). Le 
comportement reproducteur a été décrit en laboratoire chez plusieurs espèces, notamment des crevettes de grande taille (CHING et VELEZ, 1985 ; VALENTI, 1987 ; GRAZIANI et al., 1993). Les crevettes d'eau douce, comme la plupart des crustacés, portent leurs œufs jusqu'à l'éclosion qui a lieu en eau douce. Les œufs des gobies sont collés à des blocs de pierre (BELL et BROWN, 1995). Chez les crevettes les éclosions sont étalées pour chaque femelle sur plusieurs heures. Elles commencent généralement peu après la tombée de la nuit (GRAZIANI et al., 1993 ; F. HERMAN, SICA guadeloupéenne d'aquaculture, communication personnelle).

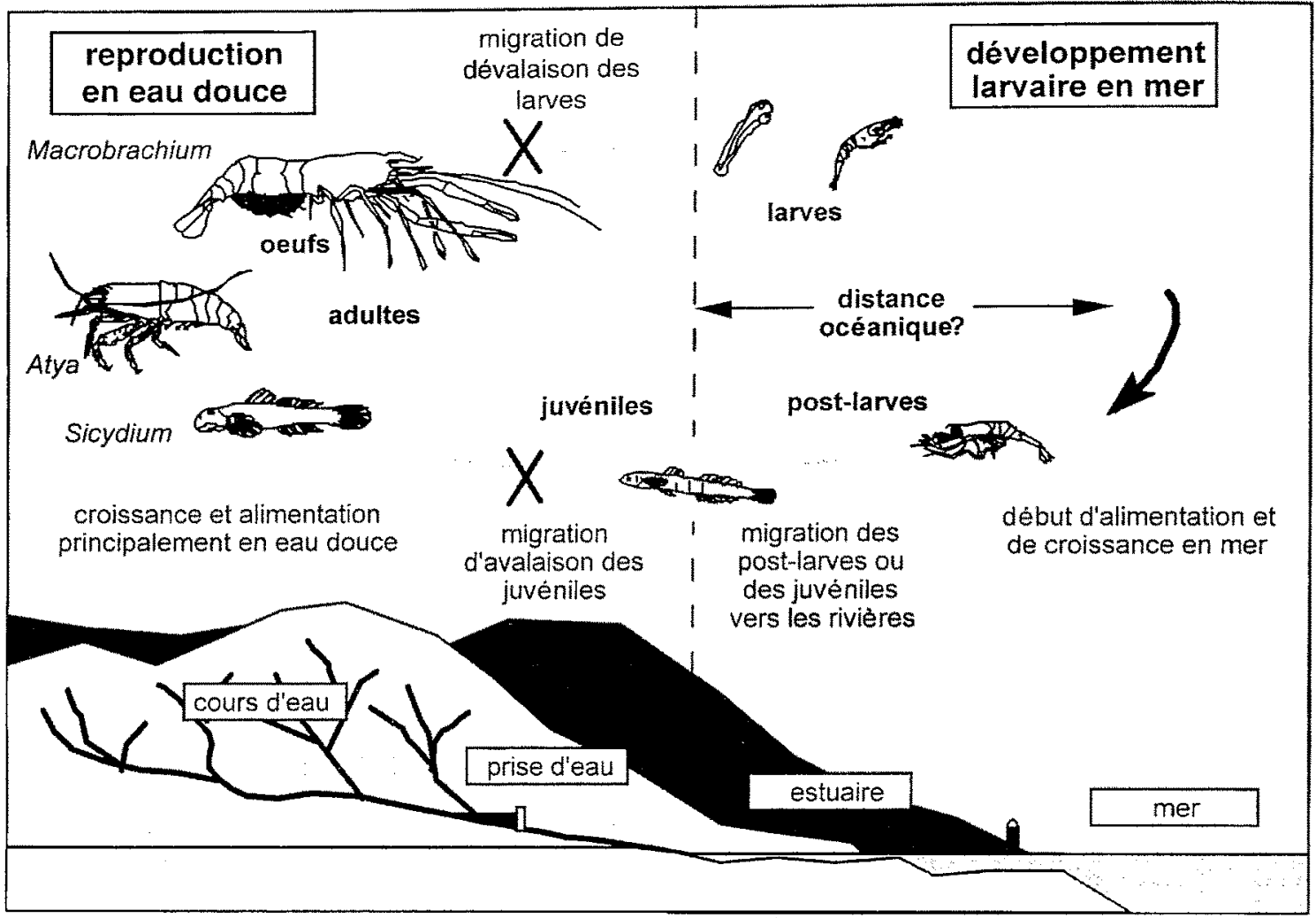

$X$ impacts des prises d'eau

\section{Figure 1}

Cycle biologique des crevettes d'eau douce et des poissons amphidromes des Antilles. Les proportions de taille entre les différents stades et groupes n'ont pas été respectées. Les tailles réelles (longueurs céphalothoraciques pour les crevettes et longueurs totales pour les poissons) sont respectivement : adulte de Sicydium $-7 \mathrm{~cm}$, adulte d'Atya $-5 \mathrm{~cm}$, adulte de Macrobrachium $-15 \mathrm{~cm}$, larve de Sicydium - 1,8 $\mathrm{mm}$, larve de Macrobrachium - $2,5 \mathrm{~mm}$, post-larve de Macrobrachium - $5 \mathrm{~mm}$, et juvénile de Sicydium - $20 \mathrm{~mm}$.

\section{Figure 1}

Life-history cycles of amphidromous freshwater shrimps and fishes in the West Indies. Outlines are out of natural proportion. Real sizes (cephalothoracic lengths for shrimps and total lengths for fishes) are : adult of Sicydium $-7 \mathrm{~cm}$, adult of Atya $-5 \mathrm{~cm}$, adult of Macrobrachium $-15 \mathrm{~cm}$, larva of Sicydium $-1.8 \mathrm{~mm}$, larva of Macrobrachium - $2.5 \mathrm{~mm}$, post-larva of Macrobrachium - $5 \mathrm{~mm}$, and juvenile of Sicydium $-20 \mathrm{~mm}$. 
La présence de juvéniles de Macrobrachium et d'Atya tout au long de l'année dans la rivière Bananier (Guadeloupe) laisse supposer un recrutement continu émanant d'une reproduction elle-même continue (Figures 2 et 3 ). Le nombre de femelles ovigères passe néanmoins par un maximum au cours des mois de juin, juillet ou aoút (Figure 3), ceci pouvant conduire à un pic maximal du nombre de juvéniles au cours des mois de septembre, octobre ou novembre (Figure 2). La reproduction est également continue chez les gobies (BELL et BROWN, 1995). Le nombre de juvéniles passant par un maximum entre juillet et septembre (THÉRÉZIEN et PLANQUETTE, 1978) et le développement larvaire nécessitant 50 à 140 jours (BELL et BROWN, 1995), un pic de reproduction centré sur les mois de mai ou de juin est probable. La présence de juvéniles de Macrobrachium et d'Atya dans les parties basses de la rivière Bananier et la présence de femelles ovigères tout au long de l'année (Figures 2 et 3) nécessitent le maintient des obstacles franchissables en permanence par les larves comme par les juvéniles.

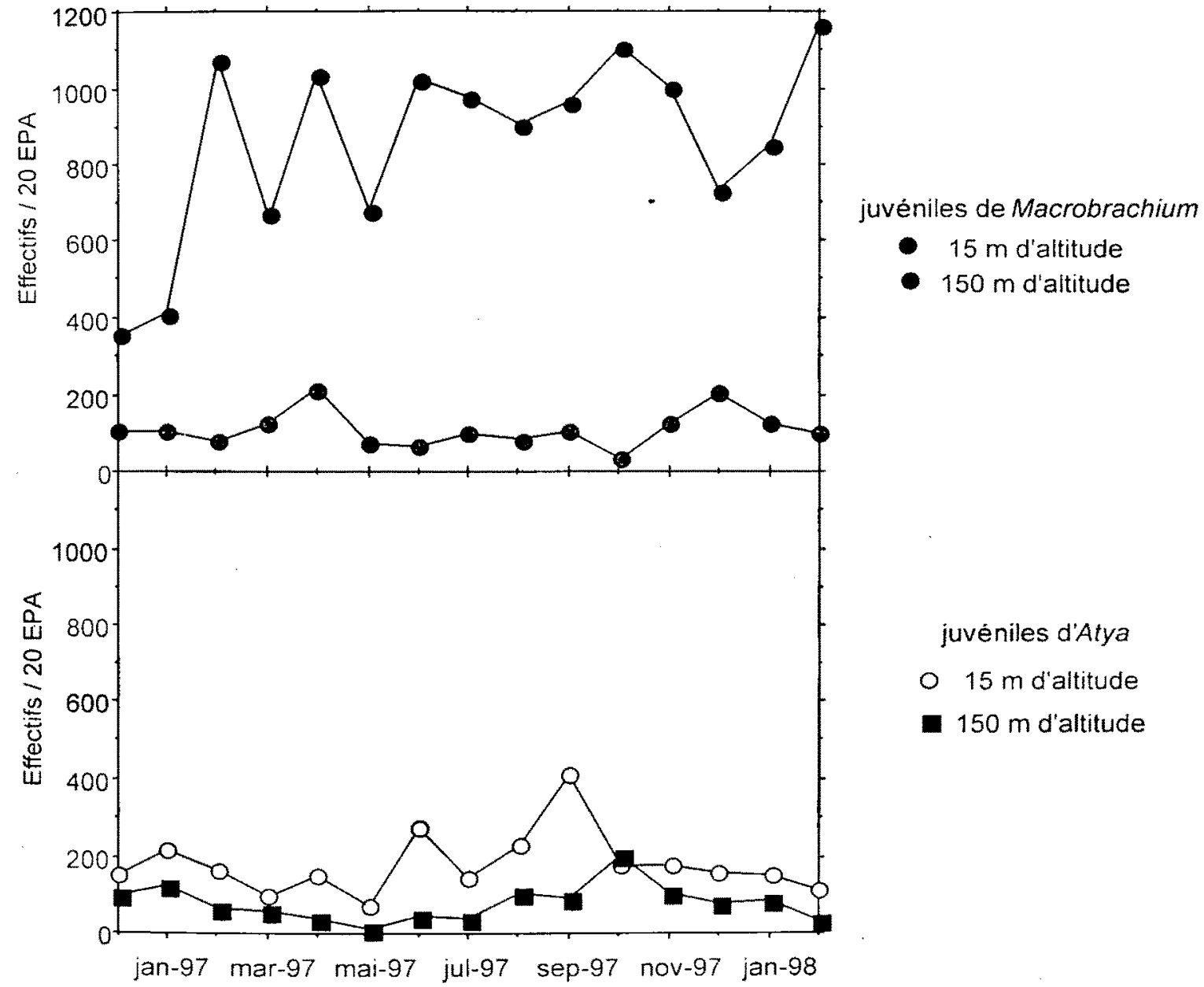

Figure 2

Variations mensuelles du nombre de juvéniles de Macrobrachium et d'Atya capturés à deux altitudes par Echantilonnage Ponctuel d'Abondance (EPA, voir FIĖVET et al., 1996 et 1999) entre décembre 1996 et février 1998, rivière Bananier, Guadeloupe.

\section{Figure 2}

Numbers of juveniles of Macrobrachium and Atya caught by Point Abundance Sampling (see FIÈVET et al., 1996 and 1999) carried out by electrofishing every month between December 1996 and February 1998 in Bananier River (Guadeloupe). 


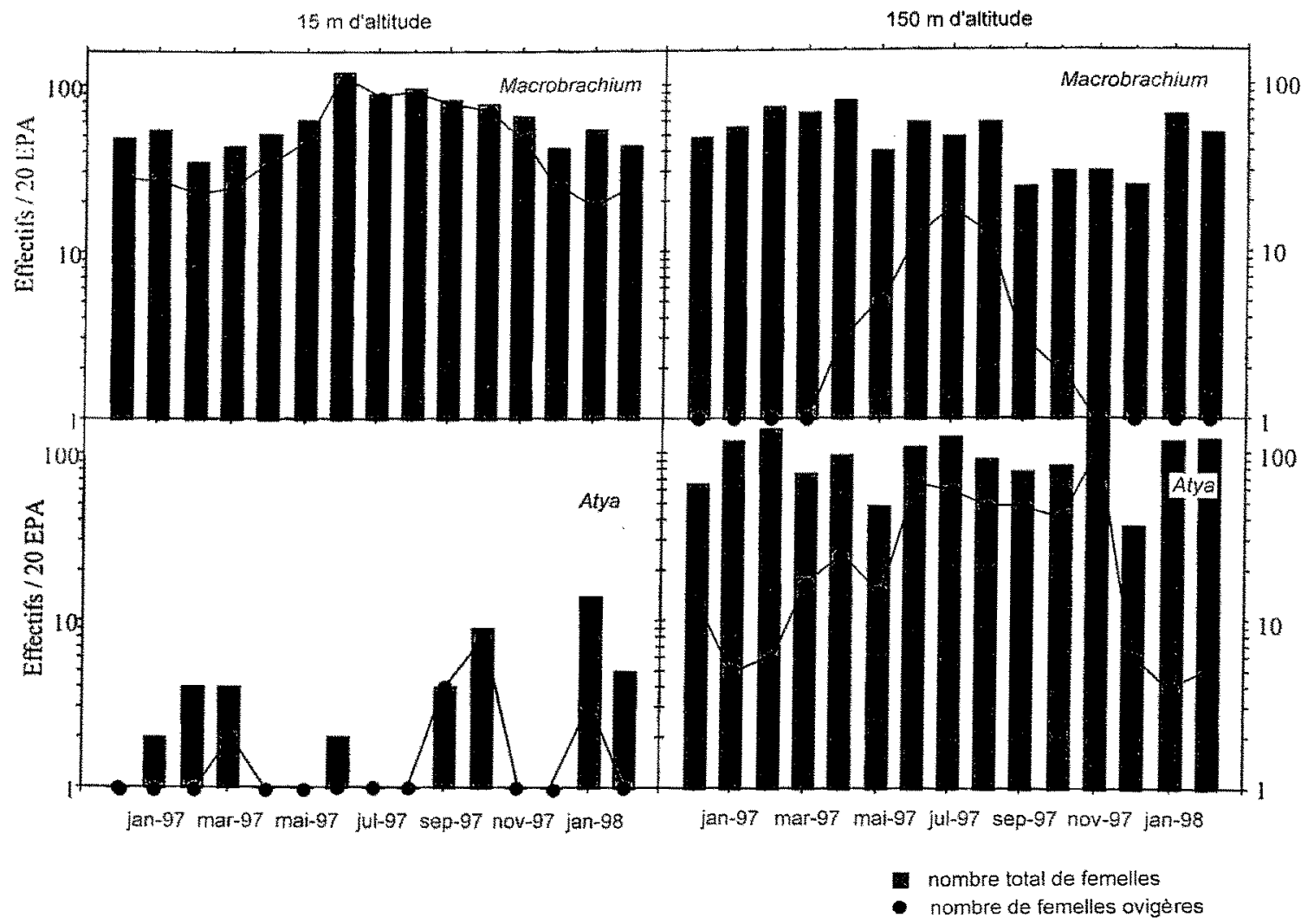

Figure 3

Variations mensuelles du nombre total de femelles et du nombre de femelles ovigères de Macrobrachium et d'Atya capturées à deux altitudes par Echantilonnage Ponctuel d'Abondance (EPA, voir FIÈVET et al., 1996 et 1999) entre décembre 1996 et février 1998, rivière Bananier, Guadeloupe.

\section{Figure 3}

Total numbers of females and numbers of ovigerous females of Macrobrachium and Atya caught by Point Abundance Sampling (see FIĖVET et al., 1996 and 1999) carried out by electrofishing every month between December 1996 and February 1998 in River Bananier (Guadeloupe).

\section{Développement larvaire}

Les larves des crevettes amphidromes passent par un grand nombre de stades zoés (plus de 8 stades) avant de devenir des post-larves (FIEVET, 1998). Les larves planctophages de Macrobrachium sont très exigeantes pour leur alimentation et n'acceptent qu'une nourriture vivante et à leur taille (des larves d'Artemia salina sont le plus souvent utilisées en élevage ; e.g., DUGAN et FRAKES, 1973 ; HERMAN et al., 1999). Ce type de nourriture étant peu abondant dans les rivières antillaises, le développement larvaire a nécessairement lieu dans les estuaires ou en mer. Les larves de certaines espèces tolèrent mieux les fortes salinités que d'autres, elles pourront donc davantage s'éloigner des côtes. La distance océanique parcourue par les larves (Figure 1) est inconnue. Elle est probablement liée à l'étendue des panaches d'eau douce ou saumâtre associés aux rivières. 


\section{PROCESSUS DE MIGRATION}

\section{Migration de dévalaison}

Une fois fécondées, les femelles de certaines espèces de crevettes, notamment Macrobrachium spp., entreprendraient une migration de dévalaison, ce qui rapprocherait les larves à naître des estuaires. L'existence d'une migration de dévalaison des femelles de Macrobrachium est soutenue par l'observation de nombreuses femelles ovigères, notamment à certaines périodes de l'année, dans les parties basses des rivières alors qu'elles sont beaucoup plus rares en altitude (Figure 3). En outre des expériences réalisées à l'aide de " chambre à courant " ont montré que les femelles ovigères de Macrobrachium acanthurus descendaient le courant contrairement aux femelles non ovigères qui avaient tendance à le remonter (HUGHES et RICHARD, 1973). Ces migrations ne sont cependant pas obligatoires et des femelles ovigères de Macrobrachium spp. ont parfois été observées en altitude, notamment lors du pic annuel de reproduction qui a lieu de mai à septembre en Guadeloupe (Figure 3). Les femelles ovigères d'autres espèces, notamment d'Atya spp., n'entreprendraient pas ce type de migration et très peu de femelles (ovigères et non ovigères) ont été observées dans les parties basses des rivières quelle que soit la période de l'année (Figure 3).

Les larves des crevettes et des gobies sont de petite taille $(<2 \mathrm{~mm})$. Elles ne peuvent donc probablement pas se maintenir dans un courant d'eau, même relativement faible (de l'ordre de $10 \mathrm{~cm} \cdot \mathrm{s}^{-1}$ ) (HANCOCK et BUNN, 1999). Des expériences menées en laboratoire ont montré que les larves de gobies se maintenaient dans la colonne d'eau par une nage active vers le haut (BELL et BROWN, 1995). Des suivis journaliers à l'aide de filets à dérive ont montré que la migration de dévalaison des larves de crevettes avait lieu principalement entre $19 \mathrm{~h}$ et $22 \mathrm{~h}$ (MARCH et al., 1998 ; BENSTEAD et al., 1999), heures qui correspondent au maximum d'éclosions. La dérive de nuit permettrait d'éviter la prédation par les poissons diurnes qui fréquentent les parties basses des rivières (e.g., Agonostomus monticola, Eleotris pisonis) (FLECKER, 1992 ; MARCH et al., 1998).

\section{Retour en rivière et migration d'avalaison}

Le retour en rivière se fait au stade post-larve. La migration d'avalaison est ensuite entreprise par les juvéniles (Figure 1). L'influence du débit d'attrait sur les crevettes et les poissons en migration tant au niveau des estuaires qu'au niveau des parties supérieures des cours d'eau n'est pas connue. La possibilité d'un " homing " - c'est-à-dire un retour des individus (post-larves) dans leur rivière d'origine et dans laquelle ils se reproduiront a plusieurs fois été évoquée pour différents genres de gobies (Sicydium, Awaous et Lentipes) mais aucun argument solide n'a été avancé pour confirmer ou infirmer cette hypothèse (ERDMAN, 1986 ; FORD et KINZIE, 1991). Chez les crevettes, la colonisation rapide des cours d'eau de tout l'archipel hawaïen par l'espèce tahitienne introduite Macrobrachium lar montre clairement que l'amphidromie limite l'isolation inter-bassin des populations et que la colonisation des rivières par les post-larves se fait au hasard (FORD et KINZIE, 1991). Dans ces conditions le débit d'eau douce au niveau des estuaires pourrait avoir un rôle majeur dans l'attractivité des post-larves par les rivières. La migration de masse d'alevins de Sicydium dans le canal de rejet $\left(1,4 \mathrm{~m}^{3} \mathrm{~s}^{1}\right)$ d'une centrale hydroélectrique en Guadeloupe (FIĖVET et Le GUENNEC, 1998) va également dans ce sens. Cet étroit canal à parois terreuses ne constitue pas a priori une zone de nursery favorable et le débit d'attrait apparaît alors comme le facteur d'attractivité prépondérant. Une comparaison in situ des quantités d'individus remontant plusieurs rivières avec des débits différents permettrait de tester l'effet attractif du débit sur les individus. 
Le rôle du débit d'attrait au niveau de la confluence de deux cours d'eau secondaires n'a pas été explicité. L'hypothèse d'une pondération de la répartition des crevettes en migration par le débit d'attrait est soutenue par des expériences de capture réalisées en Guadeloupe à l'aide de passes pièges (FIEVET, 1999a). Les individus en migration remontent en effet préférentiellement les cours d'eau principaux et seuls quelques individus remontent le faible courant d'attrait des passes pièges. Des expériences utilisant plusieurs passes pièges alimentées par différents débits d'attrait seraient néanmoins utiles pour quantifier cette relation. En outre différentes qualités d'eau issues de plusieurs rivières pourraient servir à tester l'hypothèse du homing soulevée précédemment.

Une comparaison des individus en migration capturés à l'aide de passes pièges et des individus totaux capturés par pêches électriques a montré que, pour chaque espèce, les plus petits individus migraient davantage que les plus grands (FIÈVET, 1999a). Le rhéotactisme positif, très marqué chez les juvéniles, est conservé dans une certaine mesure chez les adultes. Ceci a été confirmé par des expériences sur $M$. acanthurus à l'aide de chambre à courant (HUGHES et RICHARD, 1973). Les possibilités d'escalade des parois verticales sont également plus grandes chez les individus de petite taille. De nombreux juvéniles ont été observés en escalade sur les parois des barrages ou accumulés au pied de ces derniers (BENSTEAD et al., 1999). L'escalade est toujours effectuée sur des zones humidifiées (= "zones favorables à l'escalade "). Les gobies possèdent un organe adhésif infundibuliforme formé par la coalescence des nageoires pelviennes. Cet organe subdiscoïdal (ventouse) leur permet de se fixer au substrat et de résister aux courants les plus rapides (MAUGÉ et al., 1992). Les Sicydium mettent à profit leur adhésivité pour escalader hors de l'eau les obstacles (ERDMAN, 1986 ; FORD et KINZIE, 1991 ; FIĖVET et Le GUENNEC, 1998).

\section{IMPACTS DES BARRAGES SUR LES MIGRATIONS AMPHIDROMES}

L'ensemble des impacts potentiels sur les migrations amphidromes et la distribution des populations est présenté à la Figure 4. Les larves des crevettes et des gobies en dérive sont susceptibles d'être entraînées par les prises d'eau. En cas de prélèvement d'eau en continu, le taux de mortalité larvaire par entraînement peut être estimé en première approche par le taux de prélèvement de débit. Si 80 ou $90 \%$ du débit est prélevé. ce qui est généralement le cas en Guadeloupe (SU.MA.TEL., 1991 ; FIĖVET et Le GUENNEC, 1998), le taux de mortalité larvaire sera de 80 ou $90 \%$. Dans ces conditions la contribution relative au repeuplement de la rivière par la descendance des individus de l'amont devient très faible et la participation des individus de l'aval devient alors essentielle. En cas de perturbation anthropique à laval - pollution accidentelle entraînant une très forte mortalité par exemple - les populations de l'amont ne pourront pas être des sources importantes de repeuplement.

Dans le cas des centrales hydroélectriques, l'eau prélevée est rejetée en même temps que les larves. Le passage à travers les turbines hydrauliques soumet cependant les larves à diverses contraintes susceptibles d'entraîner des mortalités importantes : risques de chocs contre les parties fixes et mobiles des turbines, accélérations et décélérations brutales, variations très brutales de pression (CADA, 1990 ; TRAVADE et LARINIER, 1992). Diverses expériences, principalement menées sur des juvéniles de salmonidés, ont montré que les mortalités sont très variables en fonction des caractéristiques des turbines, de l'espèce et de la taille des individus (voir synthèse de LARINIER et DARTIGUELONGUE. 1989). Les mortalités sont totales dans les turbines de type Pelton dont le principe est l'envoi d'un jet d'eau à très forte vitesse sur une roue portant sur son pourtour une série d'augets en forme de double cuiller munis d'une 
échancrure centrale (LARINIER et DARTIGUELONGUE, 1989 ; TRAVADE et LARINIER, 1992). C'est malheureusement ce type de turbine qui équipe les centrales hydroélectriques de Guadeloupe (SU.MA.TEL., 1991).

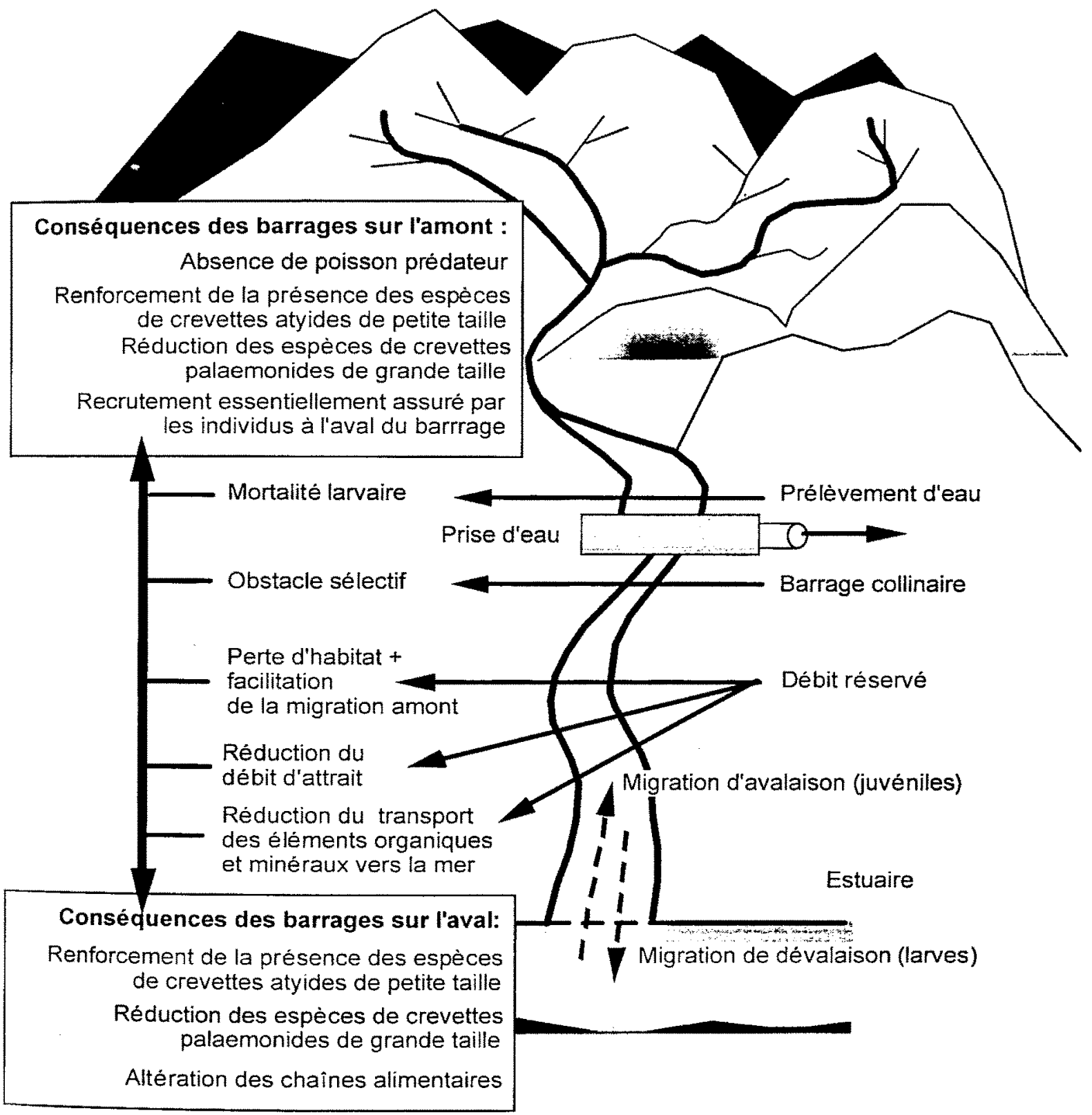

Figure 4

Impacts potentiels des barrages collinaires et des prises d'eau sur la migration amphidrome et la distribution des crevettes et de poissons des cours d'eau antillais (d'après FIĖVET, 1999c).

Figure 4

Potential impacts of low-head dams and water abstraction on amphidromous migration and distributions of freshwater shrimps and fishes of the West Indies (based on FIÈVET, 1999c). 
Concernant la migration d'avalaison, un barrage, même de faible hauteur, sans déversement d'eau constitue pour les crevettes et les gobies un obstacle infranchissable (HOLMQUIST et al., 1998). Les barrages qui possèdent un déversoir recouvert d'eau par intermittence constituent donc la plupart du temps des obstacles infranchissables. Dès que des migrateurs sont présents au niveau d'un obstacle, la libre circulation doit être assurée. En ralentissant la migration d'avalaison, d'importants taux de mortalité par prédation (BENSTEAD et al., 1999) y comprise humaine (FIEVVET et Le GUENNEC, 1998) ont parfois été enregistrés. Aussi des dispositifs de franchissements sont nécessaires pour assurer la permanence d'une libre circulation.

\section{CONCEPTION DES DISPOSITIFS DE FRANCHISSEMENTS}

En arrêtant le prélèvement d'eau durant les premières heures après le coucher du soleil BENSTEAD et al. (1999) estiment que la mortalité par entraînement des larves de crevettes dans les prises d'eau peut être réduite à $20 \%$ et moins au lieu des $80 \%$ théoriques en cas de prélèvement en continu de $80 \%$ du débit. Pour des prises d'eau en continu, les larves étant attirées par la lumière (F. HERMAN, SICA guadeloupéenne d'aquaculture, communication personnelle), des dispositifs lumineux peuvent être utilisés pour diriger les larves vers les dispositifs délivrant le débit réservé au détriment des conduites forcées (Figure 5). Par contre les juvéniles et les adultes fuient la lumière (LEE et FIELDER, 1979 ; FIEVET, 1999b). Aussi, en cas d'éclairement, il paraît indispensable que le système de prélèvement d'eau et le système de livraison du débit réservé soient suffisamment éloignés l'un de l'autre. Ils pourront par exemple être installés de part et d'autre de la rivière. La rive correspondant au système de livraison du débit réservé pourra être éclairée en amont du barrage pour attirer les larves suffisamment tôt et éviter ainsi une gêne de la migration d'avalaison. Si des résultats ponctuels ont été obtenus sur les effets répulsifs ou attractifs de la lumière chez les poissons en général, ils n'ont que rarement donné des applications en vraje grandeur en raison de leur spécificité, de leur fiabilité et de leur sensibilité aux conditions de milieu (TRAVADE et LARINIER, 1992). Des expérimentations doivent être poursuivies pour estimer les bénéfices et les inconvénients de l'utilisation de la lumière pour guider les larves des crevettes et des poissons.

Les barrages collinaires représentent des obstacles à la migration vers l'amont des individus et des espèces. Les potentialités d'escalade des juvéniles de crevettes et de Sicydium peuvent néanmoins être mises à profit pour permettre le libre franchissement de ces obstacles (FIEVET, 2000). Certaines règles doivent être néanmoins respectées. L'escalade se fait toujours près d'un écoulement d'eau (LEE et FIELDER, 1979 ; HAMANO et HAYASHI, 1992 ; FIÈVET, 1999a et b ; BENSTEAD et al., 1999). Un seul point infranchissable suffit pour que les dispositifs de franchissements soient inopérants. Les orifices noyés servant à réguler les débits constituent par exemple des zones de forts courants difficilement franchissables par les crevettes et interdisent le franchissement par escalade (FIĖVET, 2000). L'utilisation d'orifices noyés est donc à proscrire. La construction d'un canal aménagé en passe (Figure 5) n'est pas forcément nécessaire. Une simple chute d'eau peut en effet être tout aussi efficace qu'une « passe à poissons". Cette chute d'eau peut être aménagée en cascade à l'aide d'enrochements, ce qui a l'avantage de réduire l'impact visuel et d'intégrer la prise d'eau au paysage (Figure 6). Une passe en écharpe (Figure 6) permettrait la libre circulation des plus grands individus sous la chute d'eau. Son efficacité n'est cependant pas certaine et sans expérience complémentaire un couplage passe en écharpe/cascade semble être le meilleur compromis possible pour l'ensemble des individus.

La phototaxie a été utilisée au Japon pour diriger les individus en migration d'avalaison vers les dispositifs de franchissements (HAMANO et HONKE, 1997). Etant donné le rhéotactisme positif des juvéniles, il n'apparaît cependant pas indispensable de diriger les individus vers les dispositifs de franchissements si les débits s'écoulant à l'aval transitent entièrement par ces dispositifs. 


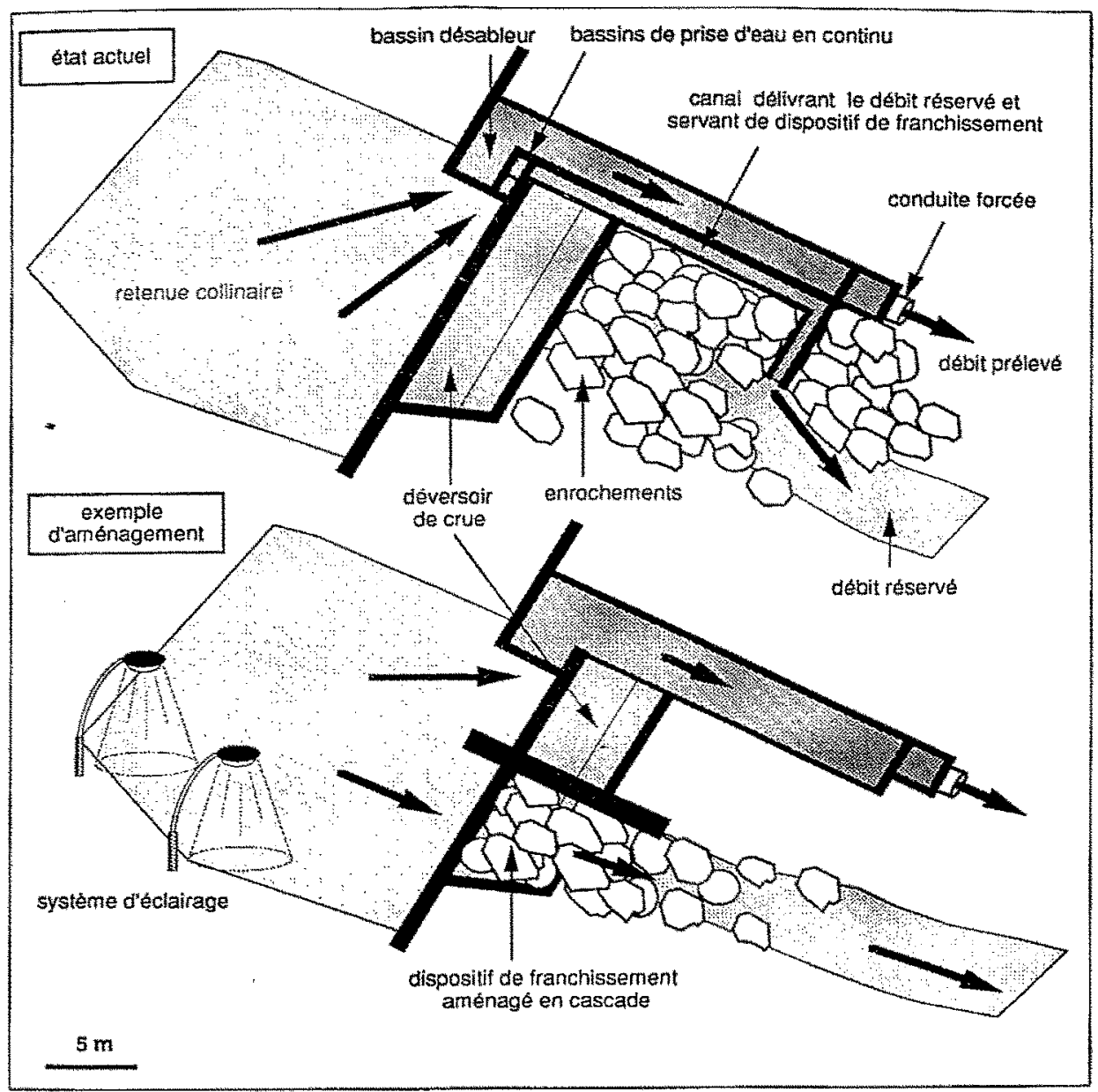

Figure 5

Vue aérienne simplifiée du dispositif de prélèvement d'eau existant à $150 \mathrm{~m}$ d'altitude sur la rivière Bananier, Guadeloupe (schéma du haut), et proposition d'aménagement (schéma du bas). Le canal délivrant le débit réservé est supposé permettre le franchissement de l'ouvrage par les individus en migration d'avalaison. Ce canal étant alimenté par des bassins de prise d'eau en continu via des orifices noyés, le dispositif est peu adapté à l'escalade des crevettes et des poissons (FIĖVT, 2000). Un aménagement plus rustique sous forme de cascade le remplacerait avantageusement. En outre aucun dispositif n'évite l'entraînement des larves en dérive dans la conduite forcée. Un système d'éclairage permettrait d'utiliser la phototaxie positive des larves pour les diriger vers le débit réservé transitant par le dispositif de franchissements plutôt que vers le débit prélevé.

Figure 5

From above scale drawings of the low-head dam at $150 \mathrm{~m}$ a.s.l. on the Bananier River, Guadeloupe (upper drawing), and proposals for improvement (lower drawing). The channel through which the compensation flow is delivered is supposed to act as a passage facility for the upstream-migrating shrimps and fishes. Because the channel is fed with water from two continuous-flow tanks with submerged oulets, this installation is not adapted to shrimp and fish climbing (FIEVET, 2000). A cascade-like pass would be more efficient. Moreover no device prevents larvae for being swept into the penstock. Lights equipment can be used to direct the larvae towards the compensation flow and to prevent them from entering the gauged flow. 


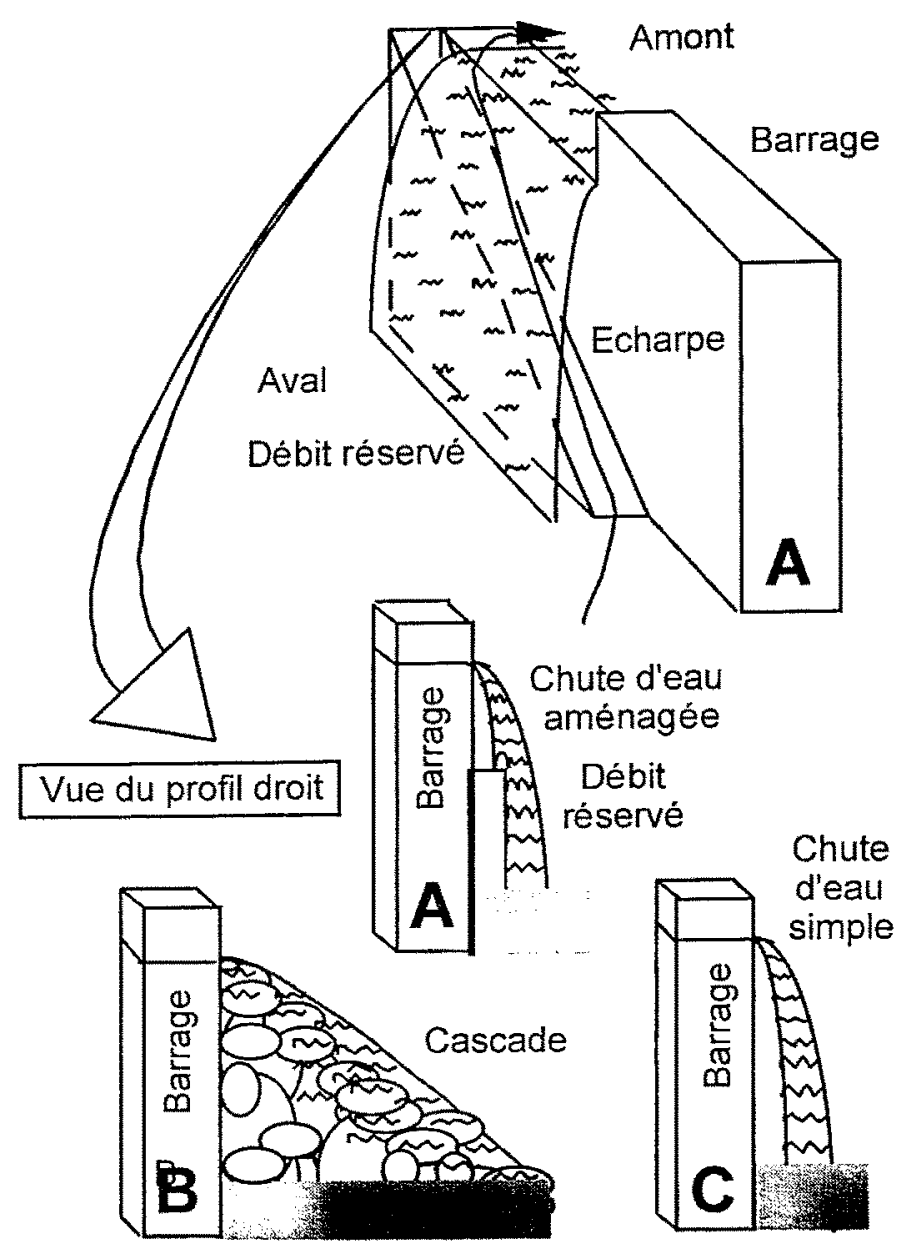

\section{Figure 6}

Proposition de dispositifs de franchissements des barrages de faible hauteur par les crevettes et les poissons amphidromes des Antilles. Une passe en écharpe (A) ou une passe aménagée en cascade (B) permettant le passage des plus grands individus seraient a priori moins sélectives qu'une simple chute d'eau (C) ne pouvant être franchie (par escalade) que par de petits individus.

\section{Figure 6}

Proposals of passage facilities in low-head dams for the amphidromous shrimps and fishes of the West Indies. Passage facilities such as " cross-side passes " $(A)$ or « cascade-like passes " $(B)$ would be less selective than a simple waterfall $(C)$ because they would allow the upstream migration of the largest individuals unlike a simple waterfall which can be climbed only by small specimens.

\section{CONCLUSION}

Le cycle biologique des espèces amphidromes implique un développement larvaire en estuaire ou en mer. La libre circulation au niveau des ouvrages est donc une condition nécessaire au maintien des populations d'amont. La continuité de la reproduction au cours de l'année implique que les obstacles soient en permanence franchissables par les larves comme par les juvéniles. La migration de dévalaison ne nécessite aucun dispositif 
particulier si de l'eau n'est pas prélevée durant les heures de dérive maximale des larves. En cas de prélèvement en continu un système d'éclairage visant à orienter les larves vers les dispositifs délivrant les débits réservés est envisageable. Son efficacité nécessite cependant une vérification sur le terrain. La migration d'avalaison peut être assurée au niveau des barrages de prise d'eau par des dispositifs sommaires de franchissements.

La libre circulation n'est cependant qu'un élément parmi d'autres à prendre en compte dans la politique de gestion des populations naturelles. La faune amphidrome est régulée en effet par de nombreux facteurs dont la dégradation de la qualité des eaux des rivières, la modification des débits naturels des cours d'eau, l'introduction d'espèces holobiotiques (Tilapias, Xiphos), la pollution des estuaires ou encore l'absence d'une réglementation de pêche spécifique aux Antilles (HOSTACHE; 1998 ; PRINGLE et SCATENA, 1999a et b). Une politique de conservation fiable ne pourra être élaborée qu'après une évaluation soigneuse de tous ces facteurs. Elle devra traiter de front tous les problèmes sous peine de voir l'un d'entre eux devenir limitant et ruiner tous les efforts consentis par ailleurs.

\section{REMERCIEMENTS}

Ce travail a été réalisé à la demande du Parc National de la Guadeloupe (contrat CNRS 0711094) et de la DIREN Guadeloupe (convention d'étude 97/EAU 001). Un financement complémentaire lors de la préparation du manuscrit a été assuré par le Ministère de l'Éducation Nationale, de l'Enseignement Supérieur et de la Recherche. La rédaction du manuscrit a bénéficié des conseils de relecture de $M$. Henri Tachet (Univ. Lyon I) et de deux examinateurs anonymes. Toute cette aide est grandement remerciée.

\section{BIBLIOGRAPHIE}

BAUCHOT M.-L., 1959. La faune ichtyologique des eaux douces antillaises. C. R. Soc. Biogéogr., 312, 7-26.

BELL K.N.I., BROWN J.A., 1995. Active salinity choice and enhanced swimming endurance in 0 to 8 -d-old larvae of diadromous gobies, including Sicydium punctatum (Pisces), in Dominica, West Indies. Mar. Biol., 121, 409-417.

BENSTEAD J.P., MARCH J.G., PRINGLE C.M., SCATENA F.N., 1999. Effects of a low-head dam and water abstraction on migratory tropical stream biota. Ecol. Appl, 9, 656-668.

CADA G.F., 1990. A review of studies relating to the effects of propeller-type turbine passage on fish early life stages. N. Am. J. Fish. Manage., 10, 418-426.

CHACE F.A.Jr., 1992. On the classification of the Caridea (Decapoda). Crustaceana, 63, 70-80.

CHACE F.A.Jr., HOBBS H.H.Jr., 1969. The freshwater and terrestrial decapod crustaceans of the West Indies with special reference to Dominica. U.S. Nat. Mus. Bull., 292, 1-258.

CHING C.A., VELEZ M.J., 1985. Mating, incubation and embryo number in the freshwater prawn Macrobrachium heterochirus (Wiegmann, 1836) (Decapoda, Palaemonidae) under laboratory conditions. Crustaceana, 49, 42-48.

COVICH A.P., MCDOWELL W.H., 1996. The stream community. In : REAGAN D.P., WAIDE R.B. (Eds), The food web of a tropical rain forest, Univ, of Chicago Press, Chicago, 433-459. 
DUGAN C.C., FRAKES T.A., 1973. Culture of brackish-freshwater shrimp Macrobrachium acanthurus, M. carcinus and M. ohione. Proc. World Maricult. Soc., 3, 185-191.

ERDMAN B.S., 1986. The green goby, Sicydium plumieri, in Puerto Rico. Trop. Fish Hob., $34,70-74$.

FIÈVET E., 1998. Distribution et capacités d'expansion des crevettes d'eau douce de la région caraïbe : exemple des genres Macrobrachium et Atya (Crustacea : Caridea). Biogeographica, 74, 1-22.

FIËVET E., 1999a. An experimental survey of freshwater shrimp upstream migration in an impounded stream of Guadeloupe Island, Lesser Antilles. Arch. Hydrobiol., 144, 339-355.

FIĖVET E., 1999b. Daylight migration of freshwater shrimp (Decapoda, Caridea) over a weir during water release from the impoundment. Crustaceana, 72, 351-356.

FIĖVET E., 1999c. Crevettes (Decapoda : Caridea) et poissons amphidromes des cours d'eau aménagés de Guadeloupe : exemples de relation dynamique aval-amont. Thèse de doctorat, Univ. Lyon I, 285 p.

FIÈVET E., 2000. Passage facilities for diadromous freshwater shrimps (Decapoda, Caridea) in the Bananier River, Guadeloupe, West Indies. Regul. Riv., 16, 101-112.

FIĖVET E., Le GUENNEC B., 1998. Migration de masse de Sicydium spp. dans les rivières de Guadeloupe : implications pour le schéma hydraulique des mini-centrales hydroélectriques « au fil de l'eau ». Cybium, 22, 293-296.

FIĖVET E., TITO De MORAIS L., TITO De MORAIS A., 1996. Quantitative sampling of freshwater shrimps : comparison of two electrofishing procedures in a Caribbean stream. Arch. Hydrobiol., 138, 273-287.

FIĖVET E., BONNET-ARNAUD P., MALLET J.-P., 1999. Efficiency and sampling bias of electrofishing for freshwater shrimp and fish in two Caribbean streams, Guadeloupe Island. Fish. Res., 44, 149-166.

FLECKER A.S., 1992. Fish predation and the evolution of invertebrate drift periodicity: evidence from neotropical streams. Ecology, 73, 438-448.

FORD J.I., KINZIE R.A.III., 1991. Life crawls upstream. Nat. Hist., 12, 61-66.

GILLET C., 1983. Les peuplements de poissons et de crevettes des rivières de la Guadeloupe : quelques données sur la biologie, la reproduction, la répartition des espèces. Rev. Hydrobiol. Trop., 16, 327-340.

GRAZIANI C.A., CHUNG K.S., DE DONATO M., 1993. Comportamentio reproductivo y fertilidad de Macrobrachium carcinus (Decapoda : Palaemonidae) en Venuezela. Rev. Biol. Trop., 41, 657-665.

GRÉGOIRE D., 1991. Etude socio-économique de la pêche en rivière dans la zone périphérique du Parc National de la Guadeloupe. Rap. Univ. Bordeaux I / Parc National de la Guadeloupe, 40 p. + annexes, rapport non publié.

HAMANO T., HAYASHI K.-I., 1992. Ecology of an atyid shrimp Caridina japonica (De Man, 1892) migrating to upstream habitats in the Shiwagi Rivulet, Tokushima Prefecture. (In Japanese with an English abstract). Researches on Crustacea, 21, 1-13.

HAMANO T., HONKE K., 1997. Control of the migrating course of freshwater amphidromous shrimps by lighting. (In Japanese with an English abstract). Crustacean Res., 26, 162-171.

HANCOCK M.A., BUNN S.E., 1999. Swimming response to water current in Paratya australiensis Kemp, 1917 (Decapoda, Atyidae) under laboratory conditions. Crustaceana, 72, 313-323.

HERMAN F., FIÈVET E., BOUCHER P., 1999. Potentialités et intérêts de l'élevage larvaire de la crevette d'eau douce indigène Macrobrachium carcinus (L.) (Palaemonidae) aux Antilles françaises. Bull. Fr. Pêche Piscic., 352, 81-90. 
HOLMQUIST J.F., SCHMIDT-GENGENBACH J.M., YOSHIOKA B.B., 1998. High dams and marine-freshwater linkages : effects on native and introduced fauna in the Caribbean. Cons. Biol., 12, 621-630.

HOSTACHE G., 1992. La vie dans les eaux douces de la Guadeloupe - poissons et crustacés. INRA et Parc National de la Guadeloupe (Eds), INRA Publ., Guadeloupe, $84 \mathrm{p}$.

HOSTACHE G., 1998. Réflexions sur la gestion des eaux douces dans le cadre de l'élaboration du schéma directeur d'aménagement du parc national de Guadeloupe : préserver le bas... pour conserver le haut, courrier de I'Environnement de l'INRA, 34, 115-118.

HUGHES D.A., RICHARD J.D., 1973. Some current-directed movements of Macrobrachium acanthurus (Wiegmann, 1836) (Decapoda, Palaemonidae) under laboratory conditions. Ecology, 54, 927-929.

HUNTE W., 1978. The distribution of freshwater shrimps (Atyidae and Palaemonidae) in Jamaica. Zool. J. Linn. Soc., 64, 135-150.

JONES C.G., LAWTON J.H., SHACHAK M., 1994. Organisms as ecosystem engineers. Oikos, 69, 373-386.

LARINIER M., DARTIGUELONGUE J., 1989. La circulation des poissons migrateurs : le transit à travers les turbines des installations hydroélectriques. Bull. Fr. Pêche Piscic., 312-313, 1-90.

LEE C.L., FIELDER D.R., 1979. A mass migration of the freshwater prawn, Macrobrachium australiense Holthuis, 1950 (Decapoda, Palaemonidae). Crustaceana, 37, 219-222.

LIEBHERR J.K. (ed.), 1988. Zoogeography of Caribbean insects. Cornell Univ. Press, Ithaca, $284 \mathrm{p}$.

LIM P., DAUBA F., SEGURA G., AUSCHER F., 1997. Peuplement de poissons de huit rivières pérennes de la Martinique. Cybium, suppl, 21, 35-46.

MARCH J.G., BENSTEAD J.P., PRINGLE C.M., SCATENA F.N., 1998. Migratory drift of larval freshwater shrimps in two tropical streams, Puerto Rico. Freshw. Biol., 40, 261-273.

MAUGE A., MARQUET G., LABOUTE P., 1992. Les Sicydiinae (Gobiidae) des eaux douces de la Polynésie française - description de trois espèces nouvelles. Cybium, $16,213-231$.

MCDOWALL R.M., 1998. Fighting the flow: downstream-upstream linkages in the ecology of diadromous fish faunas in West Coast New Zealand rivers. Freshw. Biol., 40, 111-122.

PORCHER J.P., TRAVADE F., 1992. Les dispositifs de franchissement : bases biologiques, limites et rappels réglementaires. Bull. Fr. Pêche Piscic., 326-327, 5-14.

PRINGLE C.M., 1996. Atyid shrimps (Decapoda: Atyidae) influence the spatial heterogeneity of algal communities over different scales in tropical montane streams, Puerto Rico. Freshw. Biol., 35, 125-140.

PRINGLE C.M., 1997. Exploring how disturbance is transmitted upstream : going against the flow. J. N. Am. Benthol. Soc., 16, 425-438.

PRINGLE C.M., BLAKE G.A., 1994. Quantitative effects of atyid shrimp (decapoda : Atyidae) on the depositional environment in a tropical stream : use of electricity for experimental exclusion. Can. J. Fish. Aquat. Sc., 51, 1443-1450.

PRINGLE C.M., HAMAZAKI T., 1997. Effects of fishes on algal response to storms in a tropical stream. Ecology, 78, 2432-2442. 
PRINGLE C.M., SCATENA F.N., 1999a. Aquatic ecosystem deterioration in Latin America and the Caribbean. In : HATCH L.U. and SWISHER M.E. (Eds), Managed ecosystems, the Mesoamerican experience, Oxford Univ. Press, Oxford, 104-113.

PRINGLE C.M., SCATENA F.N., 1999b. Freshwater resource development, case studies from Puerto Rico and Costa Rica. In : HATCH L.U. and SWISHER M.E. (Eds), Managed ecosystems, the Mesoamerican experience, Oxford Univ. Press, Oxford, 114-121.

PRINGLE C.M., BLAKE G.A., COVICH A.P., BUZBY K.M., FINLEY A., 1993. Effects of omnivorous shrimp in a montane tropical stream : sediment removal, disturbance of sessile invertebrates and enhancement of understory algal resources. Oecologia, 93, 1-11.

STARMÜLHNER F., THÉRÉZIEN Y., 1982a. Résultats de la mission hydrobiologique austro-française de 1979 aux îles de la Guadeloupe, de la Dominique et de la Martinique (Petites Antilles). I - Etude générale de la Guadeloupe. Rev. Hydrobiol. Trop., 15, 131-150.

STARMÜLHNER F., THÉRÉZIEN Y., 1982b. Résultats de la mission hydrobiologique austro-française de 1979 aux îles de la Guadeloupe, de la Dominique et de la Martinique (Petites Antilles). II - Etude générale de la Dominique et de la Martinique. Rev. Hydrobiol. Trop., 15, 325-345.

STATZNER B., FIÈVET E., CHAMPAGNE J.Y., MOREL R., HEROUIN E., 2000. Crayfish as geomorphic agents and ecosystem engineers : biological behavior affects sand and gravel erosion in experimental streams. Limnol. Oceanogr., 45, 1030-1040.

SU.MA.TEL., 1991. Aménagement hydro-électrique des rivières du Grand-Carbet et du Pérou, chute du Carbet Aval. Dossier de demande d'autorisation préfectorale. Société de Surveillance, Maintenance et Télésignalisation, Alberville, France.

TRAVADE F., LARINIER M., 1992. La migration de dévalaison : problèmes et dispositifs. Bull. Fr. Pêche Piscic., 326-327, 165-176.

THÉRÉZIEN Y., PLANQUETTE P., 1978. Faune ichtyologique et carcinologique des eaux douces des Antilles françaises. Notes dactylographiées du Centre de Recherches Agronomiques des Antilles et de la Guyane, INRA Guadeloupe, rapport non publié, $24 \mathrm{p}$.

VALENTI W.C., 1987. Comportamento reprodutivo de camarões de água doce. Anais de Etologia, 5, 195-202.

YOUNG G.J., DOOGE J.C.I., RODDA J.C., 1994. Global water resource issues. Cambridge Univ. Press, Cambridge. 